\title{
Manipulating the Orbital Angular Momentum of Light at the Micron Scale with Nematic Disclinations in a Liquid Crystal Film
}

\author{
Charles Loussert, Ulysse Delabre, and Etienne Brasselet* \\ Laboratoire Ondes et Matière d'Aquitaine, Université de Bordeaux, CNRS, UMR 5798, F-33400 Talence, France
}

(Received 29 March 2013; published 16 July 2013)

\begin{abstract}
We report on the experimental manipulation of the orbital angular momentum of light by exploiting a kind of topological defects that spontaneously appear in nematics—disclinations-as microscopic optical spin-orbit interfaces whose operating wavelength can be controlled electrically. Using six different kinds of disclinations, we demonstrate the efficient generation of both scalar and vectorial singular light beams with a broad topological diversity from a fundamental Gaussian beam.
\end{abstract}

DOI: 10.1103/PhysRevLett.111.037802

PACS numbers: 61.30.Jf, 42.50.Tx, 42.79.-e, 42.81.Gs

Soft matter systems are often associated with selfassembling capabilities, a representative example being liquid crystal defects [1]. A few years ago, the topological features of the local average molecular orientation of nematic liquid crystals - the director-around the defects were shown to enable the manipulation of the orbital degree of freedom of light by its polarization state via the spin-to-orbital optical angular momentum conversion phenomenon [2]. Since then, a wide range of classical or quantum applications have emerged in optics [3].

The most common methods used to realize such optical "spin-orbit" interfaces rely on either bulk [4,5] or surface $[6,7]$ photoalignment techniques of nematogenic films. This allows the director to be controlled while preserving a constant birefringent phase retardation $\Delta$. Following original designs [2], the fabricated films have an azimuthal dependence of angle $\psi$, which defines the director in plane $(x, y)$, of the form

$$
\psi=s \phi+\phi_{0},
$$

where half-integer $s$ is the strength of the defect, $\phi$ is the azimuthal angle, and $\phi_{0}$ is a constant. Under on-axis normal illumination by a circularly polarized light beam which has an axisymmetric intensity profile and the condition $\Delta=(2 n+1) \pi$ with $n$ integer, the following manipulation on light (in a quantum picture) is achieved, at least within the thin film approximation [8],

$$
\Psi_{\sigma, \ell} \rightarrow \Psi_{-\sigma, \ell+2 \sigma s}
$$

In Eq. (2), $\Psi_{\sigma, \ell}=|\sigma, \ell\rangle$ refers to a photon state with components of the spin and orbital angular momenta along the propagation direction $S_{z}=\sigma \hbar$ and $L_{z}=\ell \hbar$, respectively, with $\sigma= \pm 1$ and $\ell$ integer. Number $\ell$ is called the topological charge and refers to an azimuthal dependence of the field amplitude of the form $e^{i \ell \phi}$ [9].

However, the abovementioned liquid crystal patterning technologies do not provide photoinscribed orientational patterns with high spatial resolution. The expected material topology is indeed typically lost below $100 \mu \mathrm{m}$ [10]. This prevents realizing microscopic optical spin-orbit interfaces that are yet desirable for the development of "singular" integrated photonics, namely, the management of optical singularities at small scale.

In contrast, the use of natural liquid crystal topological defects, whose obtention does not require machining techniques, offers drastic downsizing abilities. In particular, the realization of micron-size spin-orbit optical vortex generators has been reported using nematic droplets [11]. However, so far, natural [11], electrical [12], optical $[13,14]$, or electro-optical [15] strategies based on the spontaneous creation of defects all correspond to the case $|s|=1$. Following Eq. (2), the manipulation of orbital number $\ell$ is therefore restricted to changes every two units. Moreover, an optimal spin-to-orbital angular momentum conversion efficiency, that is to say $\Delta=(2 n+1) \pi$, has only been reported in [12].

Here we unlock previous limitations of liquid crystal microscopic optical spin-orbit interfaces. We report on nearly $100 \%$ efficient conversion of Gaussian light beams into scalar and vectorial singular ones with a broad topological diversity and electrically tunable operating wavelength. This is done by exploiting the so-called Schlieren texture that spontaneously appears in nematic films with degenerate planar anchoring conditions. Such textures indeed exhibit disclinations, i.e., points around which the in-plane director field locally satisfies Eq. (1). In the present case, this is illustrated in Fig. 1.

Preparation of disclinations with various strengths.-In apolar nematics, Schlieren textures usually exhibit disclinations with strength $|s|=1 / 2$ and $|s|=1$ since higherstrength ones imply a prohibitive increase in the elastic free energy [16]. In fact, for usual nematic compounds, disclinations with strength $|s|>1$ are never observed. In contrast, as shown in Fig. 1, we have obtained highstrength disclinations with $|s|=3 / 2$ that are generally stable for a few days at room temperature. This is possible 


\begin{tabular}{ll|l|l|l|l|l|l|}
\hline$s=-3 / 2$ & $s=-1$ & $s=+1 / 2$ & \\
$\longrightarrow$
\end{tabular}

FIG. 1. Upper row: Observation of disclinations with strength $s=m / 2, \quad-3 \leq m \leq 3$ between crossed linear polarizers oriented along the $x$ and $y$ axes. Bottom row: Experimental reconstruction of the director field whose local orientation is represented by segments. Scale bar is $10 \mu \mathrm{m}$.

by doping the standard 4'- $n$-pentyl-4-cyanobiphenyl (5CB) nematic material with the nonmesogenic molecular compound named leucoquinizarin (CAS No. 17648-03-2, from Sigma-Aldrich). Such a choice is inspired by a work by Madhusudana and Pratibha, who reported, three decades ago, the observation of high-strength disclinations using different thermotropic nematics and higher working temperature [17]. Note that the existence and stability of higher-order disclinations have already been addressed theoretically $[18,19]$ and remain an open issue.

In practice, a $1 \mathrm{wt} \%$ solution of leucoquinizarin in $5 \mathrm{CB}$ is prepared following several heating and cooling cycles (passing through the nematic-isotropic transition temperature) and sonication. The mixture in its isotropic phase is inserted by capillary filling into a sandwiched cell made of glass substrates with transparent ITO electrodes, which were previously cleaned with ethanol and distilled water and then dried during $15 \mathrm{~min}$ at $80^{\circ} \mathrm{C}$. The film thickness is controlled by using a $6-\mu \mathrm{m}$-thick Mylar spacer and cells are sealed before use. Renewed textures are obtained on demand with additional temperature cycles. The structural characterization of samples is then performed at room temperature.

A qualitative visual inspection can readily be carried out. First, we note that $4|s|$ black brushes originate from a disclination with strength $s$ when observed between crossed linear polarizers, as shown in the upper panel of Fig. 1. As for the sign of $s$, it can be assessed by rotating the pair of polarizers: ensuing same and opposite rotation sense of the brushes indicates $s>0$ and $s<0$, respectively. Quantitatively, the spatial distribution of the director field is retrieved from polarimetric analysis. The main axes of the output light field under circularly polarized illumination are indeed oriented at $45^{\circ}$ from the local optical axis, i.e., the director. The reconstructed director patterns for $s=m / 2$ with $-3 \leq m \leq 3$ are displayed in the bottom panel of Fig. 1. Finally, since uniform intensity distribution is observed between crossed circular polarizers (not shown here), except near the core of the disclinations, a spatially uniform birefringent retardation $\Delta$ is assumed in what follows.

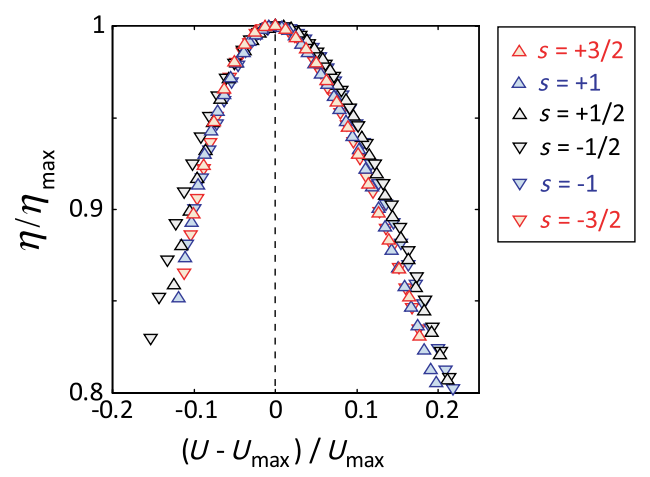

FIG. 2 (color online). Reduced spin-to-orbital angular momentum efficiency $\eta / \eta_{\max }$ versus reduced applied voltage difference, $\left(U-U_{\max }\right) / U_{\max }$, for scalar optical vortex generation using six different kinds of disclinations. In all cases, $U_{\text {max }} \approx 1 V_{\text {rms. }}$

Generation of scalar vortex beams.-Scalar (labeled with $S$ ) optical vortex beams refer to linear superpositions of states of the form

$$
\Psi_{\ell}^{\mathrm{S}}(a, b)=a \Psi_{-1, \ell}+b \Psi_{+1, \ell},
$$

where $a$ and $b$ are constant coefficients, with $|a|^{2}+|b|^{2}=1$. They refer to elliptically polarized vortex beams with topological charge $\ell$ that carries an averaged spin and orbital angular momentum per photon $S_{z}=\left(|b|^{2}-|a|^{2}\right) \hbar$ and $L_{z}=\ell \hbar$, respectively. Since the polarization state of light can easily be controlled by means of polarization optics, we restrict our demonstration to $(a, b)=(1,0)$ or $(0,1)$. From Eq. (2) this occurs when an incident light beam with state $\Psi_{ \pm 1,0}$ impinges on a disclination with strength $s= \pm \ell / 2$ and $\Delta=(2 n+1) \pi$.

However, in general, the birefringent retardation of the sample at rest does not satisfy the condition required by Eq. (2). This can nevertheless be adjusted to the operating wavelength by electrical means. Indeed, since the used nematic has a positive dielectric anisotropy at low frequency (here, $1 \mathrm{kHz}$ ), the application of a voltage $U$ between the two electrodes tends to realign the director along $z$, hence reducing $\Delta$.

In our experiments, we use a circularly polarized $\mathrm{TEM}_{00}$ beam from a He-Ne laser operating at $632.8 \mathrm{~nm}$ wavelength. The beam divergence angle is $\theta_{0} \simeq 0.7^{\circ}$ and the beam spot radius is typically set in the range 10-100 $\mu \mathrm{m}$ by varying the location of the sample along the $z$ axis. The electrical tuning of the spin-to-orbital angular momentum conversion efficiency is monitored by measuring the power

TABLE I. Maximal values of the spin-to-orbital angular momentum conversion efficiency $\eta_{\max }$ for scalar optical vortex generation with six different kinds of disclinations.

\begin{tabular}{lcccccc}
\hline \hline$s$ & $-3 / 2$ & -1 & $-1 / 2$ & $+1 / 2$ & +1 & $+3 / 2$ \\
\hline$\eta_{\max }$ & $94.5 \%$ & $98.9 \%$ & $99.1 \%$ & $99.0 \%$ & $99.2 \%$ & $94.2 \%$ \\
\hline \hline
\end{tabular}




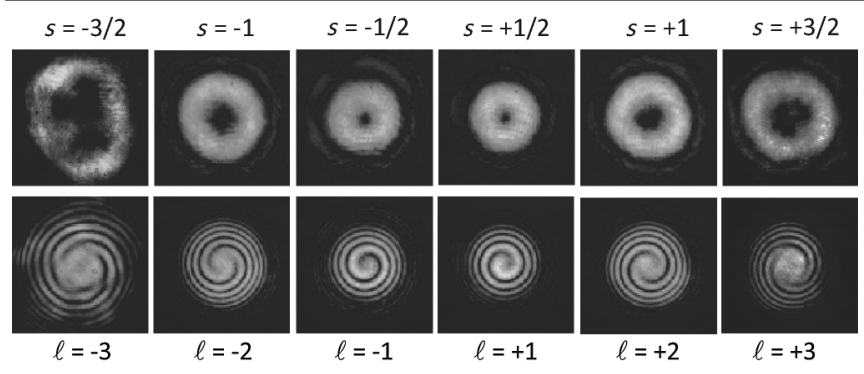

FIG. 3. Generation of scalar vortex beams from disclinations with strength $s=m / 2,-3 \leq m \leq 3$ and fixed incident circular polarization state; here, $\sigma=+1$. Upper row: Output intensity profiles. Bottom row: Interference patterns that result from the coaxial superposition of the vortex beam with a reference Gaussian beam having the same polarization state.

fraction $\eta$ of the incident beam that is converted into an optical vortex beam, namely, the power of the contracircularly polarized component of the output light field. The results are shown in Fig. 2, where the values of $\eta\left(U=U_{\max }\right)=\eta_{\max }$ are displayed in Table I.

We thus conclude on the possible generation of almost pure states $\Psi_{\ell= \pm 1, \pm 2, \pm 3}^{\mathrm{S}}$, which can easily be extended to a broad range of wavelengths by adjusting the applied voltage. Typical results are displayed in Fig. 3 that exhibits the characteristic doughnut-shaped intensity profiles. In addition, the helical distribution of the phase around the on-axis optical phase singularity is retrieved from the interference of the generated vortex states with a coaxial reference Gaussian beam having the same polarization state. Spiraling fringed patterns with $|\ell|$ spiral arms are indeed observed whereas their handednesses are associated with the sign of $\ell$.

More quantitatively, we note that the radius of maximal intensity $R_{\ell}$ increases with $\ell$ as the Laguerre-Gaussian modes $\mathrm{LG}_{0, \ell}$ do. We indeed observe a dependence of the form $R_{\ell} \propto|\ell|^{1 / 2}$, as shown in Fig. 4(a). The expected local azimuthal dependence of the phase around the vortex core,
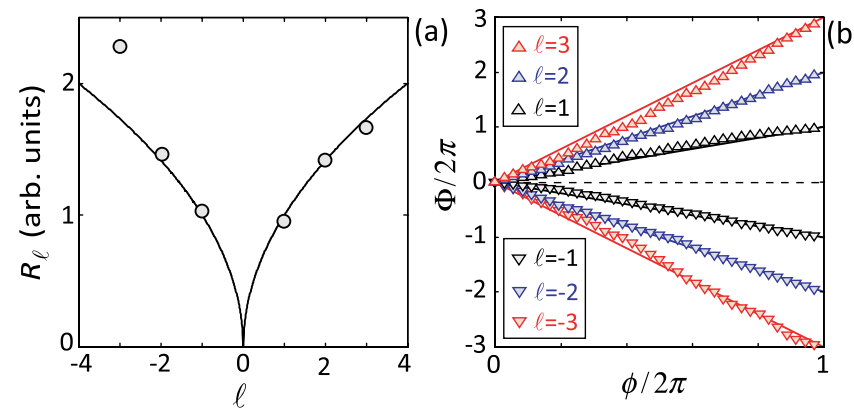

FIG. 4 (color online). Intensity and phase characterization of the scalar optical vortices shown in Fig. 3. (a) Radius of maximal intensity of the transverse intensity profiles. Solid line refers to the Laguerre-Gaussian behavior of the form $R_{\ell} \propto|\ell|^{1 / 2}$. (b) Azimuthal behavior of the phase profiles. Solid lines refer to linear dependence of the form $\Phi=\ell \phi$.

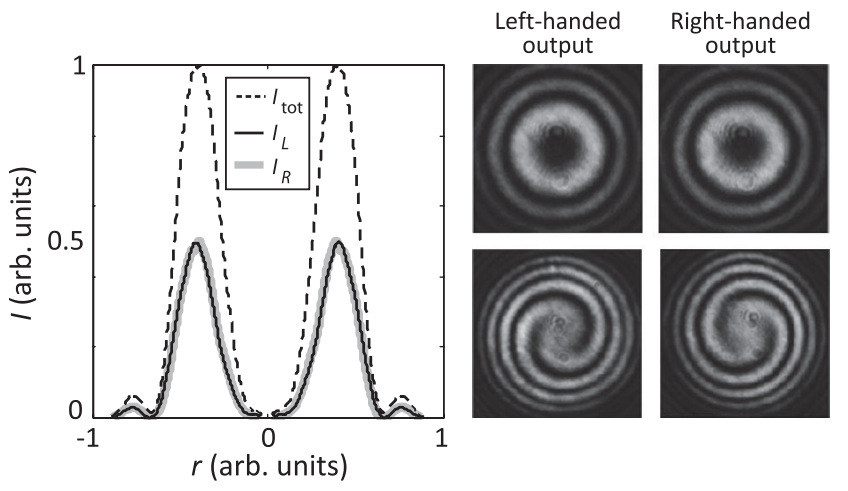

FIG. 5. Intensity and phase analyses for the states content of a vector beam defined by state $\Psi_{2}^{\mathrm{V}}$. Left: Azimuthally averaged radial intensity profiles of the total and left- or right-handed circular components of the output light fields, $I_{\text {tot }}$ and $I_{L, R}$. Right: Upper panels show the spatial light intensity distributions $I_{L, R}$ whereas bottom ones show the interferograms obtained from the coaxial superposition of the left or right circular components with a reference Gaussian beam having the same polarization state.

$\Phi=\ell \phi$, is also retrieved experimentally as shown in Fig. 4(b). These results are obtained following the Stokes polarimetry procedure used in [11], namely, $\Phi=$ $\arctan \left[\left(I_{+45^{\circ}}-I_{-45^{\circ}}\right) /\left(I_{0^{\circ}}-I_{90^{\circ}}\right)\right]$, where $I_{\alpha}$ refers to the transverse spatial intensity distribution of the linearly polarized component of the output light field oriented at an angle $\alpha$ from the $x$ axis.

Generation of vector beams. - Vector $(V)$ beams refer to the following superposition of states

$$
\Psi_{\ell}^{\mathrm{V}}=\frac{\Psi_{-1, \ell}+\Psi_{+1,-\ell}}{\sqrt{2}}
$$

Such beams carry zero averaged spin and orbital angular momentum per photon, $S_{z}=L_{z}=0$. From Eq. (2), vector beams can be obtained when a $x$-linearly polarized incident state $\left(\Psi_{-1,0}+\Psi_{+1,0}\right) / \sqrt{2}$ impinges on a disclination with strength $s=\ell / 2$ and $\Delta=(2 n+1) \pi$, the latter condition being adjusted electrically as discussed above. The particular case $s=+1$ is illustrated in Fig. 5. In this figure, the intensity and phase characteristics of the individual states that constitute $\Psi_{2}^{\mathrm{V}}$ are shown, namely, right- and

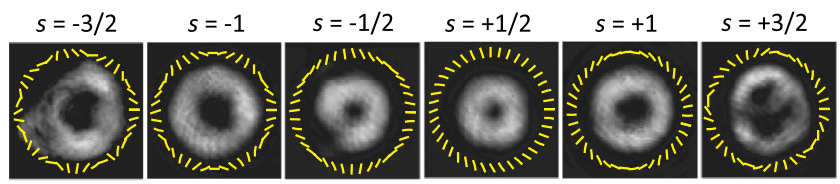

FIG. 6 (color online). Generation of various vector beams by using six different disclinations presented in Fig. 1 for an incident fundamental Gaussian beam linearly polarized along $x$ axis. The segments superimposed to the total intensity profiles refer to polarization azimuth patterns, taken at radius of average maximal intensity. 
TABLE II. Weighted average of the reduced third Stokes parameter and of the ratio between the minor and major axes of the polarization ellipse $\left\langle s_{3}\right\rangle$ and $\langle e\rangle$, respectively, for the vectorial vortex states $\Psi_{\ell= \pm 1, \pm 2, \pm 3}^{\mathrm{V}}$ obtained from six different disclinations with strength $s=m / 2,-3 \leq m \leq 3$.

\begin{tabular}{lcccccc}
\hline \hline$s$ & $-3 / 2$ & -1 & $-1 / 2$ & $+1 / 2$ & +1 & $+3 / 2$ \\
\hline$\left\langle s_{3}\right\rangle$ & 0.29 & 0.14 & 0.22 & 0.24 & 0.20 & 0.27 \\
$\langle e\rangle$ & 0.15 & 0.07 & 0.11 & 0.12 & 0.10 & 0.14 \\
\hline \hline
\end{tabular}

left-handed scalar optical vortices with topological charge \pm 2 and equal intensity distributions.

A particular feature of states $\Psi_{\ell}^{\mathrm{V}}$ is associated with a well-defined inhomogeneous polarization state. More precisely, the light field is linearly polarized everywhere while its orientation is given by the unit vector $\mathbf{p}_{\ell}(\phi)=$ $\cos (\ell \phi) \mathbf{x}+\sin (\ell \phi) \mathbf{y}$. Experimentally, this is verified by full-Stokes polarimetric analysis of the total field emerging from the sample. Typical results are displayed in Fig. 6 where polarization azimuth patterns $\mathbf{p}_{\ell}(\phi)$ superimposed to the total intensity profiles correspond to the radius of average maximal intensity, for six different disclinations with strength, $s=m / 2$ with $-3 \leq m \leq 3$. Figures of merit of the generated states can also be assessed quantitatively. To this purpose we introduce (i) $\left\langle s_{3}\right\rangle$ as the weighted average of the reduced third Stokes parameter $s_{3}=\left(I_{L}-I_{R}\right) /\left(I_{L}+I_{R}\right)$, namely, $\left\langle s_{3}\right\rangle=$ $\iint s_{3}(x, y) I_{\text {tot }}(x, y) d x d y / \iint I_{\text {tot }}(x, y) d x d y$, and (ii) $\langle e\rangle$ as the weighted average of ratio $e$ between the minor and major axes of the polarization ellipse, namely, $\langle e\rangle=$ $\iint e(x, y) I_{\mathrm{tot}}(x, y) d x d y / \iint I_{\mathrm{tot}}(x, y) d x d y$. The measured values are reported in Table II. Since an ideal state $\Psi_{\ell}^{\mathrm{V}}$ corresponds to $\left\langle s_{3}\right\rangle=0$ and $\langle e\rangle=0$, we conclude on a fair quality of the generated vector states.

To conclude, here we have reported on the electrically tunable topological shaping of light using disclinations of various strengths up to $|s|=3 / 2$ in nematic liquid crystal films doped with a nonmesogenic molecular compound. The generation of various kinds of singular beams has been experimentally demonstrated, namely, (i) scalar optical vortex beams carrying on-axis optical phase singularity and (ii) vector beams with singular distribution of the polarization state azimuth angle. In both cases, this has been demonstrated over a broad range of integer topological charges, namely, $-3 \leq \ell \leq 3$. These results unlock previous vortex field topological charge limitations of microscopic self-engineered optical spin-orbit interfaces made of liquid crystal topological defects, so far restricted to $|\ell|=2$. This brings a complete toolbox for the manipulation of optical orbital angular momentum at the micron scale, which is not yet possible with other existing strategies based on liquid crystals. Future challenges could be to further enrich and control the topological diversity of three-dimensional orientational architectures of liquid crystals. Regarding this issue, we notice that we have also observed defects with higher strength, which are, however, less stable than the ones reported here. To promote possible applications, it might be worth considering the photopolymerization of liquid crystal defects, since this would allow us to realize perennial singular micro-optical elements while preserving their electrical tunability.

We acknowledge financial support from DGA Contract No. 201160083.

*e.brasselet@loma.u-bordeaux1.fr

[1] I. Dierking, Textures of Liquid Crystals (Wiley-VCH, Weinheim, 2003).

[2] L. Marrucci, C. Manzo, and D. Paparo, Phys. Rev. Lett. 96, 163905 (2006).

[3] L. Marrucci, E. Karimi, S. Slussarenko, B. Piccirillo, E. Santamato, E. Nagali, and F. Sciarrino, J. Opt. 13, 064001 (2011).

[4] S. C. McEldowney, D. M. Shemo, R. A. Chipman, and P. K. Smith, Opt. Lett. 33, 134 (2008).

[5] S. Nersisyan, N. Tabiryan, D. M. Steeves, and B. R. Kimball, Opt. Express 17, 11926 (2009).

[6] S.-W. Ko, Y.-Y. Tzeng, C.-L. Ting, A. Y.-G. Fuh, and T.-H. Lin, Opt. Express 16, 19643 (2008).

[7] S. Slussarenko, A. Murauski, T. Du, V. Chigrinov, L. Marrucci, and E. Santamato, Opt. Express 19, 4085 (2011).

[8] E. Nagali, F. Sciarrino, F. D. Martini, B. Piccirillo, E. Karimi, L. Marrucci, and E. Santamato, Opt. Express 17, 18745 (2009).

[9] L. Allen, M.W. Beijersbergen, R. J.C. Spreeuw, and J. P. Woerdman, Phys. Rev. A 45, 8185 (1992).

[10] D. Mawet, E. Serabyn, K. Liewer, C. Hanot, S. McEldowney, D. Shemo, and N. O'Brien, Opt. Express 17, 1902 (2009).

[11] E. Brasselet, N. Murazawa, H. Misawa, and S. Juodkazis, Phys. Rev. Lett. 103, 103903 (2009).

[12] E. Brasselet and C. Loussert, Opt. Lett. 36, 719 (2011).

[13] E. Brasselet, Phys. Rev. A 82, 063836 (2010).

[14] B. Yang and E. Brasselet, J. Opt. 15, 044021 (2013).

[15] R. Barboza, U. Bortolozzo, G. Assanto, E. VidalHenriquez, M. G. Clerc, and S. Residori, Phys. Rev. Lett. 109, 143901 (2012).

[16] S. Chandrasekhar, Liquid Crystals (Cambridge University Press, Cambridge, England, 1992), 2nd ed.

[17] N. V. Madhusudana and R. Pratibha, Mol. Cryst. Liq. Cryst. 103, 31 (1983).

[18] V. A. Raghunathan, Europhys. Lett. 24, 479 (1993).

[19] H. R. Brand and H. Pleiner, Europhys. Lett. 26, 395 (1994). 